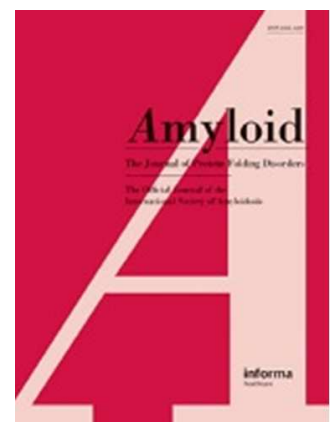

\title{
Epidemiology of ATTRV30M neuropathy in Cyprus and the modifier effect of complement C1q on the age of disease onset
}

\begin{tabular}{|c|c|}
\hline Journal: & Amyloid \\
\hline Manuscript ID & DAMY-2018-0086.R2 \\
\hline Manuscript Type: & Original Article \\
\hline Date Submitted by the Author: & 17-Sep-2018 \\
\hline Complete List of Authors: & $\begin{array}{l}\text { Andreou, Savanna; Cyprus Instirute of Neurology and Genetics, Clinic A } \\
\text { Panagiotou, Elena; Cyprus Instirute of Neurology and Genetics, Clinic A } \\
\text { Michaelidou, Kyriaki; Cyprus Instirute of Neurology and Genetics, Electron } \\
\text { Microscopy/Molecular Pathology } \\
\text { Pirpa, Panayiota; Cyprus Instirute of Neurology and Genetics, Electron } \\
\text { Microscopy/Molecular Pathology } \\
\text { Hadjisavvas, Andreas; Cyprus Instirute of Neurology and Genetics, Electron } \\
\text { Microscopy/Molecular Pathology } \\
\text { El Salloukh, Adonis; Saint George's Medical School, University of Nicosia } \\
\text { Barnes, Daniel; Centre for Cancer Genetic Epidemiology, Department of } \\
\text { Public Health and Primary Care, University of Cambridge } \\
\text { Antoniou, Antonis; Centre for Cancer Genetic Epidemiology, Department of } \\
\text { Public Health and Primary Care, University of Cambridge } \\
\text { Agathangelou, Petros; Cyprus Instirute of Neurology and Genetics, Clinic A } \\
\text { Papastavrou, Katia; Pantheo Eye Center } \\
\text { Christodoulou, Kyproula; Cyprus Instirute of Neurology and Genetics, } \\
\text { Neurogenetics } \\
\text { Tanteles, George; Cyprus Instirute of Neurology and Genetics, Clinical } \\
\text { Genetics Clinic } \\
\text { Kyriakides, Theodoros; Cyprus Instirute of Neurology and Genetics, Clinic A }\end{array}$ \\
\hline Keywords: & ATTRV30M, neuropathy, complement c1q, Cyprus, Epidemiology \\
\hline
\end{tabular}


2

3

4

5

6

7

8

9

10

11

12

13

14

15

16

17

18

19

20

21

22

23

24

25

27

28

29

30

31

32

33

34

35

36

37

39

40

41

42

43

44

45

46

47

48

49

50

51

52

53

54

55

56

57

58

59

60

URL: http://mc.manuscriptcentral.com/damy 


\section{Epidemiology of ATTRV30M neuropathy in Cyprus and the modifier effect of complement C1q on the age of disease onset}

Savanna Andreou ${ }^{1}$, Elena Panayiotou ${ }^{1}$, Kyriaki Michailidou ${ }^{2}$, Panayiota Pirpa ${ }^{2}$, Andreas Hadjisavvas $^{2}$, Adonis El Salloukh ${ }^{3}$, Daniel Barnes ${ }^{4}$, Antonis Antoniou ${ }^{4}$, Petros Agathangelou$^{1}$, Katia Papastavrou ${ }^{5}$, Kyproula Christodoulou ${ }^{6}$, George A. Tanteles ${ }^{7}$ and Theodoros Kyriakides ${ }^{1}$

${ }^{1}$ Department of Neuropathology/ Neurology Clinic A, The Cyprus Institute of Neurology \& Genetics, Nicosia, Cyprus.

${ }^{2}$ Department of Electron Microscopy/Molecular Pathology, The Cyprus Institute of Neurology \& Genetics, Nicosia, Cyprus.

${ }^{3}$ St. George's Medical School, Nicosia University, Nicosia, Cyprus.

${ }^{4}$ Centre for Cancer Genetic Epidemiology, Department of Public Health and Primary Care, University of Cambridge, Cambridge, UK.

${ }^{5}$ Pantheo Eye Center, Limassol, Cyprus

${ }^{6}$ Neurogenetics Department, The Cyprus Institute of Neurology \& Genetics, Nicosia, Cyprus.

${ }^{7}$ Clinical Genetics Clinic, The Cyprus Institute of Neurology \& Genetics, Nicosia, Cyprus.

Corresponding Author: Dr T.Kyriakides

Senior Consultant Neurologist

Head of Clinic A, Director of Neuropathology Lab

Professor, Cyprus School of Molecular Medicine

Cyprus Institute of Neurology and Genetics

6, International Airport Avenue

P.O Box 23462, 1683 Nicosia, Cyprus

Tel: 0035722358600

Fax: 0035722392786

E-mail: theodore@cing.ac.cy

Web site: www.cing.ac.cy 


\begin{abstract}
Background: ATTRV30M amyloidosis is a lethal autosomal dominant sensorimotor and autonomic neuropathy caused by amyloid deposition composed of aggregated misfolded TTR monomers with the V30M mutation. The age of onset in patients with ATTRV30M varies in different foci and the mechanism behind it is still unknown.
\end{abstract}

Methods: The tertiary neurology center following all ATTRV30M patients in Cyprus was used to collect demographic data to estimate; prevalence, incidence, penetrance, anticipation, time from disease onset to diagnosis and transplantation. Ocular, cardiac and leptomeningeal involvement in transplanted patients was explored. Correlation of C1q tagging SNPs with age of disease onset was carried out.

Results: Prevalence and incidence for ATTRV30M neuropathy in Cyprus are 5.4/100,000 and $0.3 / 100,000$ respectively. Mean age of onset is 40.6 years and anticipation is 8.3 years. Penetrance reaches $51 \%$ and $75 \%$ by the ages of 50 and 80 years respectively. In liver transplanted patients rates of ocular, cardiac and leptomeningeal involvement were estimated to be $60 \%, 20 \%$ and $16 \%$ respectively. C1q polymorphisms correlated with age of disease onset.

Conclusion: ATTRV30M neuropathy has a rising prevalence in Cyprus due to improved survival of patients. Late onset complications are becoming a major problem. Complement $\mathrm{C} 1 \mathrm{q}$ appears to be a modifier in this disease.

\title{
Keywords
}

Epidemiology; ATTRV30M; neuropathy; complement; C1q; Cyprus

List of Abbreviations: ATTRm: Hereditary transthyretin amyloid protein; ATTRV30M: hereditary transthyretin amyloid protein with V30M mutation; CI: confidence interval; CING: Cyprus Institute of Neurology and Genetics; CMAP: compound muscle action potential; HR: hazard ratio; HWE: Hardy-Weinberg equilibrium; LT: liver transplantation; SNAP: sensory nerve action potential; SNPs: single nucleotide polymorphisms; TFNEs: transient focal neurological episodes; TTR: transthyretin 


\section{Introduction}

Hereditary transthyretin (ATTRm) amyloidosis may give rise to a peripheral neuropathy where extracellular mutated transthyretin (TTR) is deposited, predominantly in the peripheral nerves, heart and renal tissues [1]. It is an autosomal dominant disorder caused by the substitution of valine by methionine at position 30 [2]. Generally considered to be a progressive and fatal disease with autonomic and sensorimotor symptoms. ATTRV30M neuropathy has an average survival of 10 years after diagnosis and until recently the only available treatment was orthotopic liver transplantation (LT) $[3,4]$.

The original cases of ATTRV30M neuropathy were recorded in Portugal [1], followed by reports in Sweden and Japan [5, 6] which are large endemic areas with families carrying single genetic mutations [7]. Smaller endemic foci have been reported in Majorca and Cyprus [8, 9]. Recent studies have also reported late onset ATTR V30M amyloidosis cases with distinct clinical phenotype in non-endemic areas [10]. ATTRm neuropathy is highly heterogeneous, both genetically and phenotypically [11] and ATTRV30M is the most common ATTRm neuropathy diagnosed in Portugal, Brazil and Sweden [7]. In France and Japan, around 30 different TTR mutations have been detected [12-14]. An epidemiological study performed in 2003 in the Cypriot population recorded a total of 36 patients from 22 ATTRV30M families. The average age of onset was found to be 48.6 years with the most common initial symptom being neuropathic foot pain. Also, the study reported positive anticipation independent of the transmitting parents' sex.

Penetrance and age of onset are variable among different populations. Epigenetic and genetic factors are believed to contribute to this variability. In search of modifier genes our group has examined the role of the complement pathway, since complement factors have been found to co-precipitate with amyloid in various forms of amyloidotic neuropathy [15]. Certain polymorphic sites in C1q were previously found to correlate with the age of onset in Cypriot patients suggesting that complement C1q may indeed be a modifier [16]. Data from our group suggest that C1q ablation in TTRKO/Met30 transgenic mice enhances amyloid deposition, while upregulating apoptosis and oxidative stress $[17,18]$. 
Epidemiology of this disease is evolving; patients are now diagnosed earlier and are offered more treatments. In the current study we report epidemiologic data, 15 years after the initial follow-up of the previous study [9] and we re-evaluate the impact of C1q on the age of onset in a greater cohort.

\section{Materials and Methods}

\section{Epidemiological and demographic data}

Demographic data were collected on all patients diagnosed at the Cyprus Institute of Neurology and Genetics (CING). The CING is the only tertiary neurology center in Cyprus where all patients with ATTRV30M neuropathy are referred to for molecular diagnosis and clinical management including referral for LT and other treatments. All cases of ATTRV30M neuropathy in Cyprus are strictly familial. The small size of the total population and the closeness of Cypriot families ensure complete ascertainment of symptomatic cases. The data collected for each patient or carrier included current age, sex, place of residence, place of origin, age at onset of symptoms, type of symptoms at presentation, time to diagnosis, duration of illness and age at death. Genetic counselling was also provided. Furthermore, a timeline to transplantation was also constructed based on the patients' files. The census date used for estimating prevalence and incidence of ATTRV30M amyloidosis within the population of Cyprus is 31/12/2016. Patients are defined as carriers of the V30M mutation who are symptomatic, while carriers simply have the mutation but no relevant symptoms. The patients were diagnosed by genetic testing, typical clinical phenotype and a biopsy (either rectal, abdominal fat or peripheral nerve). All transplanted patients and all patients receiving Tafamidis had to have a positive biopsy prior to treatment. In patients who have had liver transplantation (all in the UK) the results were not always available; however in 23 patients the biopsy results were available. 


\section{Neurophysiological assessment}

Sensory and sensorimotor axonal neuropathies are defined as length dependent neuropathies involving only sensory or sensory and motor axons respectively, (normal values; sural $\mathrm{SNAP}>6.0 \mu \mathrm{V}, \mathrm{CV}>44 \mathrm{~m} / \mathrm{s}$, peroneal EDP $\mathrm{CMAP}>4 \mathrm{mV}, \mathrm{CV}>42 \mathrm{~m} / \mathrm{s}$, tibial AHL CMAP $>5 \mathrm{mV}, \mathrm{CV}>41 \mathrm{~m} / \mathrm{s}$. Mild demyelination was defined as slowing of conduction velocities out of proportion to CMAP values but not fulfilling the diagnostic criteria of chronic demyelinating neuropathy. Normal conduction was defined as a result within the amplitude and conduction velocities of our lab.

\section{Leptomeningeal involvement}

Leptomeningeal involvement was diagnosed either from history of transient focal neurological episodes () and/or the presence of microhaemorrahges on T2-gradient echo sequence (GRE) magnetic resonance imaging. TFNEs were defined as transient motor and/or sensory and/or speech disturbances of variable onset and duration. Typically they did not have the instantaneous onset of transient ischemic attacks.

\section{Ocular and cardiac involvement}

The following abnormalities were considered as evidence of ocular involvement; keratoconjunctivitis sicca, abnormal conjunctival vessels, anterior chamber amyloid deposits, vitreous amyloid deposits, abnormal shape of the iris and amyloid deposits, presence of intraocular hypertension and lid abnormalities.

The following abnormalities were considered as evidence of cardiac involvement; enddiastolic interventricular thickness of $>12 \mathrm{~mm}$, reduced longitudinal function with tissue Doppler Imaging ( $\mathrm{e}^{\prime}, \mathrm{a}^{\prime}$ and $\mathrm{s}^{\prime}<5 \mathrm{~cm} /$ second and absolute longitudinal strain $<15 \%$ ), preserved radial and apical function and sparkling texture. 


\section{Candidate gene approach}

Signed informed consent from 44 patients and 14 carriers seen in the last 12 months were obtained followed by blood collection. DNA samples from 80 patients (deceased included) and 19 carriers were available to be assessed for $21 \mathrm{C} 1 \mathrm{q}$ tagging SNPs. A corresponding number of age matched controls was also assessed for the same SNPs, in order to assess their minor allele frequencies and establish Hardy-Weinberg equilibrium status. Nineteen (19) SNPs were assessed by a standardized allelic discrimination protocol using real time-PCR for all patients/carriers and the corresponding number of age matched controls. Two SNP assays rs15940 and rs17433222 (no longer industrially produced) were assessed via the conventional $\mathrm{PCR} /$ sequencing protocol. Approval from the Cyprus Bioethics Committee was obtained for the study whereby the consent forms and subsequent protocols followed were accepted (Cyprus National Bioethics Committee - ЕЕВK/ЕП /2015/36)

\section{Statistical analysis}

Age specific penetrance was estimated by a modified segregation retrospective likelihood analysis $[19,20]$ to account for the non-random ascertainment of carriers with respect to their disease phenotype, using the MENDEL software [21]. Anticipation was estimated based on the age of onset in a pool of available offspring-parent pairs and its statistical significance was tested by a paired t-test. The number of potential asymptomatic carriers was estimated from 31 families taking into account that the disease is autosomal dominant exhibiting a 50\% probability of inheritance. Statistical significance of the mean age of onset between ATTRV30M patients was evaluated via the two-sample t-test. The p-value threshold, which is used to indicate the statistical significance, was set to values lower than $0.05(\mathrm{p}<0.05)$.

Concerning SNPs analysis two different statistical tests were performed. In the first test, considering both symptomatic and asymptomatic carriers (19 asymptomatic /80 patients), the age of onset was used as the initial start point for the patients and the age of last healthy observation was used for asymptomatic carriers. The second test was performed in respect to age of onset and included only patients (80 cases). The Cox proportional 
hazards model was carried out, where the event was defined as the age of onset. Analyses were performed in $\mathrm{R}$ (coxph) using cluster analysis where variance was adjusted to account for ascertainment bias due to the fact that data was only collected from affected families.

\section{Results}

\section{Number of patients}

On the $31^{\text {st }}$ of December 2016, 46 living ATTRV30M patients were recorded in Cyprus (24 males and 22 females). Since the late 1980 s and until the $31^{\text {st }}$ of December 2016,82 patients were formally diagnosed in Cyprus with ATTRV30M neuropathy. All patients originated from 31 families, 14 families from Kyrenia, 14 from Limassol and 3 from Paphos. All patients were of Greek- Cypriot descent.

Out of the 46 living patents, 36 had been transplanted. Three patients were on TTR stabilizers and three patients had been referred to start a stabilizer. Three patients were participating in a Phase 3 trial of an anti-sense oligonucleotide and one patient was at stage III neuropathy and was receiving supportive treatment.

\section{Prevalence}

The prevalence was estimated based on the official population census of $31 / 12 / 2016$. The total population in the republic of Cyprus on that date was 854,800. 46 living ATTRV30M patients were recorded in Cyprus. Thus, the prevalence of ATTRV30M neuropathy was estimated to be $5.4 / 100,000$. Prevalence at the risk population (over the age of 18) was estimated to be $6.4 / 100,000$.

\section{Incidence}

During the period 2003-2016, 31 patients were diagnosed with ATTRV30M amyloidosis in Cyprus. The average annual incidence was estimated as $0.3 / 100,000$ of the total population. 


\section{Anticipation}

The anticipation of ATTRV30M amyloidosis was estimated based on the age of onset of 49 patient pairs of offspring-parents and was derived from a pool of 82 patients.

The mean age of onset in parents was 50.3 years and 40.7 years in offspring (p-value: $9.23 \times 10^{-7}$, paired t-test). Thus, positive anticipation was recorded, where the age of onset in the offspring was on average 9.6 years earlier than their respective affected parent. The sex of the transmitting parent did not appear to influence anticipation (p-value: 0.54, twosample t-test). Also, the anticipation for 23 offspring-parent pairs (where the offspring manifested between 2003 and 2016), was assessed. In this group the offspring were diagnosed 8.3 years earlier than their transmitting parent ( $\mathrm{p}$-value: $2.73 \times 10^{-5}$, paired $\mathrm{t}$ test).

\section{Estimation of asymptomatic carriers}

Potential carriers were identified through their re-visited pedigrees. Of the 259 potential carriers estimated, 215 were over the age of 18. Therefore, the prevalence of asymptomatic carriers was estimated to be $30.3 / 100,000$ and $30 / 100,000$ over the age of 18.

\section{Penetrance}

Fig 1a illustrates the estimated penetrance by age. It is obvious that penetrance appears to be age related. At the age of 20 years the risk of developing the disease is $6 \%$, whereas the risk at ages of 50 and 80 increases to $51 \%$ and $75 \%$ respectively.

\section{Mean age of onset}

The mean age of onset (mean \pm SD) of all patients diagnosed in Cyprus until 31/12/2016 was estimated to be $44.5 \pm 15(n=82)$, in males $43.8 \pm 16(n=40)$ and $45.2 \pm 15(n=42)$ in females (no statistical difference recorded, p-value: 0.68, two-sample t-test). The mean age of onset in live patients on 31/12/2016 was 40.6 $\pm 12(n=46)$ (Fig 1b), while in patients 
which manifested between 2003 and 2016 it was $41.4 \pm 14(n=29)$. The mean period from onset to diagnosis in live patients was 18.5 months $(n=46)$.

From the late 1980's until 31/12/2016, 53 ATTRV30M carriers were confirmed (five died asymptomatic). The mean age of live carriers $(n=48)$ was estimated $47.5 \pm 15$, which does not differ significantly (p-value: 0.27, two-sample t-test) compared to all ATTRV30M patients $(\mathrm{n}=82)$.

\section{Phenotype}

Symptoms at onset

The initial presenting symptoms of the 46 living patients are summarized in Table 1 . The most commonly reported initial symptoms were "sharp shooting" or "burning" pains (74\%) and the most commonly observed sign was dissociated sensory loss (96\%).

Neurophysiological testing at diagnosis

Nerve conduction studies at diagnosis were performed for 43 patients. Sensorimotor axonal neuropathy was detected in 25 patients (58\%), whereas 11 patients $(26 \%)$ had sensory axonal neuropathy. Only one patient $(2.3 \%)$ was diagnosed with mild demyelination and six patients (14\%) had normal nerve conduction.

Amyloid deposits at diagnosis

Biopsy results at diagnosis were available for 23 ATTRV30M patients: 14 patents $(61 \%)$ were rectal positive, two patients $(9 \%)$ rectal negative, two sural nerve positive $(9 \%)$, two abdominal fat positive $(9 \%)$, one cardiac muscle positive $(4 \%)$, one patient both rectal and abdominal fat positive (4\%) and another one was both urinary bladder and abdominal fat positive (4\%). SAP scan results were available for 21 patients: 15 were positive $(71 \%)$ and 6 were negative $(29 \%)$.

Liver transplantation 
Thirty-six from the 46 living patients underwent orthotopic liver transplantation (LT). The mean period from symptom onset to transplantation was 37.3 months and from diagnosis to transplantation was 18.6 months. Serum albumin levels at transplantation were only available for 31 patients and their estimated average was $40.7 \mathrm{~g} / \mathrm{L}$ (normal 35$50 \mathrm{~g} / \mathrm{L}$ ). All patients were in Stage I neuropathy.

Leptomeningeal involvement in transplanted patients

Six transplanted patients (17\%) displayed leptomeningeal involvement: one subclinical (positive MRI), five had TFNEs (Focal Neurological Episodes). MRI was performed in four patients without a pacemaker; two were normal and two abnormal. CT scans were performed on 10 patients but were found to be normal in all cases.

Ocular and cardiac involvement in transplanted patients

Ocular and cardiac involvement was also assessed in transplanted patients. Ocular assessment was carried out on 32 of the patients. Nineteen were found to be positive (59\%), while 13 were negative (41\%) for ocular involvement.

Twenty nine transplanted patients have had repeated echocardiography assessments and six were found to have cardiac involvement (21\%). None presented with cardiomyopathy.

\section{Candidate gene approach to assess for disease modifiers-SNPs analysis}

Eighty patients (44 alive and 36 deceased), 19 carriers- both classified as "cases" and 99 controls (wild type TTR) were analyzed. The Hardy-Weinberg equilibrium (HWE) was calculated for all SNPs in the control population (Supplementary Table 1). SNPs rs186037 and rs294223 did not follow the Hardy-Weinberg equilibrium (HWE p-value threshold: 0.05) and were thus excluded from further analysis.

Using both asymptomatic and symptomatic carriers (19 asymptomatic/80 patients) and by using age of onset as the time point for the patients and the age of last healthy observation for the asymptomatic carriers we performed Cox proportional hazards 
analysis with the event being the age of onset or the age of last healthy observation. We performed the analyses in $\mathrm{R}$ (coxph), estimating the cluster robust variance on family to account for the fact that we have data from relatives. The hazard ratio (HR) and confidence interval (CI) can be potentially biased as we do not take into account the nonrandom ascertainment of the samples in the study. The results below assess the modifying effect of each SNP to the disease. HR was calculated in respect to the major allele (Supplementary Table 2).

In the first statistical test, the most significant modifying effect association came from rs672693 (p-value: $5.87 \times 10^{-3}$, HR: 1.63 , 95\% CI: $1.15-2.30$ ) whereas the two SNPs (rs9434, rs294180) correlated strongly with age of onset while rs665691 and rs12126436 were also moderately associated. Therefore, allele G of rs672693 increases the risk of manifesting ATTRV30M amyloidosis earlier.

In respect to the age of onset when using patients only ( 80 cases) the most significant modifying effect came from rs665691 (p-value: $1.87 \times 10^{-3}, \mathrm{HR}: 1.54, \mathrm{CI}: 1.17-2.03$ ) and the others that were significantly associated with it. Thus, earlier age of onset is associated with allele $\mathrm{C}$ of rs665691 in the Cypriot patients. The entire squared correlation coefficient table amongst the tested SNPs in the carriers' pool can be found in supplementary table 3 .

\section{Discussion}

Prevalence in other endemic areas such as Northern Portugal, Northern Sweden and Nagano, Japan is estimated to be 163.1 in 100,000, 104 in 100,000 and 1.29 in 100,000 population respectively [22] Also, the age of onset varies across different endemic areas. The mean age of onset in Portuguese and Japanese ATTRm patients is 33 years [23, 24], while in Swedish patients the mean age of onset is 56 years of age [25]. Disease anticipation has also been reported in most endemic countries such as Portugal, Sweden and Japan [26-29]

The currently estimated prevalence of ATTRV30M neuropathy in Cyprus is 5.4/100,000, compared to the 2003 value of $3.7 / 100,000$ [9]. This increase in prevalence is accounted 
for by the increase in the number of living patients. New manifesting carriers are diagnosed and referred for LT earlier and survive longer. Currently, there are 46 living patients compared to the 27 included in the previous study. Also, increased awareness within the families themselves facilitates earlier diagnosis. The average incidence recorded between 2003 and 2016 appears to be lower $(0.3 / 100,000)$ compared to the 1992-2002 average $(0.7 / 100,000)$. This is may be partly due to a larger pool of undiagnosed patients that were diagnosed during 1992-2002.

Penetrance of the disease may also impact the incidence assuming a continuous pool of carriers. The biological basis of penetrance is poorly understood and both by genetic and environmental factors are involved. In the current study, we estimated the penetrance of ATTRV30M neuropathy in Cyprus to be $51 \%$ and $75 \%$ at the ages of 50 and 80 years respectively. In France, $18 \%$ and $85 \%$ respectively, $69 \%$ and $85 \%$ in Portugal [30] and $11 \%$ and $69 \%$ in Sweden [28].

The mean age of symptom onset was $41.4 \pm 14$ years compared to $48.6 \pm 15$ years in the previous survey. Additionally, we confirm that the patients' sex does not influence the age of onset in Cypriot patients [9], as similarly observed in the Swedish population [23]. This is in contrast to both the Portuguese and Brazilian populations where the patients' sex does influence the age of onset [26,31].

Anticipation was found to remain unchanged and may explain the approximately 8 year reduction in the age of onset in the current study, although earlier diagnosis is possible. Mean period from symptom onset to diagnosis was 18.5 months, largely due to patient denial, failure of non-specialists to recognize the disease, as well as the criterion for diagnosis at the CING where the presence of dissociated sensory loss is required.

The phenotype of the patients in both surveys followed the typical pattern of small fiber and autonomic neuropathy. Also, no patients with demyelinating neuropathy were identified (most common ATTRV30M misdiagnosis). It is our current practice to diagnose the presence of ATTRV30M neuropathy when dissociated sensory loss is present on clinical examination. In all patients tissue confirmation of amyloidosis was 
required. Leptomeningeal involvement was present in about $16 \%$ of the post transplanted patients, who also had ocular amyloid disease.

The genetic analysis has found that C1q SNPs rs665691, rs158761, rs172378, rs672693, rs9434 and rs294180 correlate with an earlier age of disease onset. C1q activates the classical complement pathway ultimately producing the potent $\mathrm{C} 5 \mathrm{a}$ anaphylatoxin, which in turn activates phagocytic cells and induces clearance of TTR amyloid deposits [32].

SNP rs665691 (allele C) was found to be one of the most significant associations. According to dbSNP-NCBI, rs665691 is found among microRNA 6127 (MIR6127), which is located on $1 \mathrm{p} 36.12$. Interestingly, this SNP is also found to be significantly associated $(\mathrm{P}=0.0006)$ with the risk of developing rheumatoid arthritis in a cohort of 822 patients and over 1000 controls [33].

SNP rs672693 (allele G) was also to have a significant modifying effect in the test involving the combined TTRV30M carrier analysis, although it was also found to be significant in the analysis including only patients. SNP rs672693 is located within an intronic region of $\mathrm{ClqC}$ chain and may be involved in splicing. Polymorphisms within intronic regions may alter splicing and gene transcription [34].

Genetic variants of other complement components have previously been shown to act as modifiers of both the onset and the expression of ATTRm amyloidosis, more specifically the $\mathrm{C} 3 \mathrm{~F}$ and $\mathrm{C} 4 \mathrm{~A} 3$ variants were found to be significantly higher in early onset patients [35].

The correlation of C1q polymorphisms and age of onset of ATTRV30M neuropathy may or may not be a causative association. There are some human and animal data that provide evidence consistent with a pathogenic role for complement in amyloid neuropathy. Nerve biopsies of patients with amyloidotic neuropathy show coprecipitation of complement with amyloid $[15,36]$. C1q ablation in the ATTRV30M neuropathy mouse model exacerbates amyloid deposition while complement C5a agonists stimulate phagocytosis and reduce amyloid deposits [18, 32]. It is therefore likely the C1q polymorphisms have a causative association with age of onset. 
Furthermore, studies in lupus and rheumatoid arthritis have shown that the expression levels of $\mathrm{Clq}$ and other complement components significantly change depending on the polymorphism expressed, thus affecting the overall function of the complement cascade $[37,38]$.

In the current study an increased prevalence of 5.4/100,000 for ATTRV30M was recorded in Cyprus, mainly accounted for by improved survival due to LT. We have confirmed that the phenotype of the disease remains largely that of the typical lengthdependent sensorimotor and autonomic neuropathy. No sporadic cases were recorded. We confirm the presence of positive anticipation of about 8 years and an age specific penetrance different from other endemic foci. Additionally, we have demonstrated a number of C1q tagging SNPs associated with age of onset supporting a modifier role for $\mathrm{C} 1 \mathrm{q}$ which need to be replicated in other populations.

Disclosure statement: The authors report no conflicts of interest.

Funding: This work was supported by Telethon Cyprus and Pfizer's GLOBAL Aspire Grants.

\section{References}

[1] Andrade C. A peculiar form of peripheral neuropathy; familiar atypical generalized amyloidosis with special involvement of the peripheral nerves. Brain. 1952;75:408-27.

[2] Saraiva MJ, Birken S, Costa PP, et al. Amyloid fibril protein in familial amyloidotic polyneuropathy, Portuguese type. Definition of molecular abnormality in transthyretin (prealbumin). J Clin Invest. 1984;74:104-19.

[3] Conceicao I, De Carvalho M. Clinical variability in type I familial amyloid polyneuropathy (Val30Met): comparison between late- and early-onset cases in Portugal. Muscle Nerve. 2007;35:116-8.

[4] Koike H, Tanaka F, Hashimoto R, et al. Natural history of transthyretin Val30Met familial amyloid polyneuropathy: analysis of late-onset cases from non-endemic areas. J Neurol Neurosurg Psychiatry. 2012;83:152-8.

[5] Andersson R. Familial amyloidosis with polyneuropathy. A clinical study based on patients living in northern Sweden. Acta Med Scand Suppl. 1976;590:1-64.

[6] Araki S, Mawatari S, Ohta M, et al. Polyneuritic amyloidosis in a Japanese family. Arch Neurol. 1968;18:593-602. 
[7] Plante-Bordeneuve V, Said G. Familial amyloid polyneuropathy. Lancet Neurol. 2011;10:1086-97.

[8] Reines JB, Vera TR, Martin MU, et al. Epidemiology of transthyretin-associated familial amyloid polyneuropathy in the Majorcan area: Son Llatzer Hospital descriptive study. Orphanet J Rare Dis. 2014;9:29.

[9] Dardiotis E, Koutsou P, Papanicolaou EZ, et al. Epidemiological, clinical and genetic study of familial amyloidotic polyneuropathy in Cyprus. Amyloid. 2009;16:32-7.

[10] Koike H, Misu K, Ikeda S, et al. Type I (transthyretin Met30) familial amyloid polyneuropathy in Japan: early- vs late-onset form]. Arch Neurol. 2002;59:1771-6.

[11] Reilly MM, Adams D, Booth DR, et al. Transthyretin gene analysis in European patients with suspected familial amyloid polyneuropathy. Brain. 1995;118:849-56.

[12] Araki S, Ando Y. Transthyretin-related familial amyloidotic polyneuropathy-Progress in Kumamoto, Japan (1967-2010). Proc Jpn Acad Ser B Phys Biol Sci. 2010;86:694-706.

[13] Plante-Bordeneuve V, Lalu T, Misrahi M, et al. Genotypic-phenotypic variations in a series of 65 patients with familial amyloid polyneuropathy. Neurology. 1998;51:708-14.

[14] Ikeda S, Takei Y, Tokuda T, et al. Clinical and pathological findings of non-Val30Met TTR type familial amyloid polyneuropathy in Japan. Amyloid. 2003;1:39-47.

[15] Hafer-Macko CE, Dyck PJ, Koski CL. Complement activation in acquired and hereditary amyloid neuropathy. Journal of the peripheral nervous system : JPNS. 2000;5:131-9.

[16] Dardiotis E, Koutsou P, Zamba-Papanicolaou E, et al. Complement C1Q polymorphisms modulate onset in familial amyloidotic polyneuropathy TTR Val30Met. J Neurol Sci. 2009;284:158-62.

[17] Pisalyaput K, Tenner AJ. Complement component C1q inhibits beta-amyloid- and serum amyloid P-induced neurotoxicity via caspase- and calpain-independent mechanisms. J Neurochem. 2008;104:696-707.

[18] Panayiotou E, Fella E, Papacharalambous R, et al. C1q ablation exacerbates amyloid deposition: A study in a transgenic mouse model of ATTRV30M amyloid neuropathy. PloS one. 2017;12:e0175767.

[19] Antoniou AC, Sinilnikova OM, Simard J, et al. RAD51 135G-->C modifies breast cancer risk among BRCA2 mutation carriers: results from a combined analysis of 19 studies. Am J Hum Genet. 2007;81:1186-200.

[20] Barnes DR, Lee A, Investigators E, et al. Evaluation of association methods for analysing modifiers of disease risk in carriers of high-risk mutations. Genet Epidemiol. 2012;36:274-91. 
[21] Lange K, Weeks D, Boehnke M. Programs for Pedigree Analysis: MENDEL, FISHER, and dGENE. Genet Epidemiol. 1988;5:471-2.

[22] Schmidt $\mathrm{HH}$, Waddington-Cruz $\mathrm{M}$, Botteman MF, et al. Estimating the global prevalence of transthyretin familial amyloid polyneuropathy. Muscle Nerve. 2018;57:829-837.

[23] Sousa A, Andersson R, Drugge U, et al. Familial amyloidotic polyneuropathy in Sweden: geographical distribution, age of onset, and prevalence. Hum Hered. 1993;43:288-94.

[24] Ikeda S, Nakazato M, Ando $Y$, et al. Familial transthyretin-type amyloid polyneuropathy in Japan: clinical and genetic heterogeneity. Neurology. 2002;58:1001-7.

[25] Holmgren G, Costa PM, Andersson C, et al. Geographical distribution of TTR met30 carriers in northern Sweden: discrepancy between carrier frequency and prevalence rate. J Med Genet. 1994;31:351-4.

[26] Sousa A, Coelho T, Barros J, et al. Genetic epidemiology of familial amyloidotic polyneuropathy (FAP)-type I in Povoa do Varzim and Vila do Conde (north of Portugal). Am J Med Genet. 1995;60:512-21.

[27] Drugge U, Andersson R, Chizari F, et al. Familial amyloidotic polyneuropathy in Sweden: a pedigree analysis. J Med Genet. 1993;30:388-92.

[28] Hellman U, Alarcon F, Lundgren $\mathrm{HE}$, et al. Heterogeneity of penetrance in familial amyloid polyneuropathy, ATTR Val30Met, in the Swedish population. Amyloid. 2008;15:181-6.

[29] Yamamoto $\mathrm{K}$, Ikeda S, Hanyu N, et al. A pedigree analysis with minimised ascertainment bias shows anticipation in Met30-transthyretin related familial amyloid polyneuropathy. J Med Genet. 1998;35:23-30.

[30] Plante-Bordeneuve V, Carayol J, Ferreira A, et al. Genetic study of transthyretin amyloid neuropathies: carrier risks among French and Portuguese families. J Med Genet. 2003;40.

[31] Bittencourt PL, Couto CA, Clemente C, et al. Phenotypic expression of familial amyloid polyneuropathy in Brazil. Eur J Neurol. 2005;12:289-93.

[32] Fella E, Sokratous K, Papacharalambous R, et al. Pharmacological stimulation of phagocytosis enhances amyloid plaque clearance; evidence from a transgenic mouse model of ATTR neuropathy. Front Mol Neurosci. 2017;10.

[33] Trouw LA, Daha N, Kurreeman FA, et al. Genetic variants in the region of the C1q genes are associated with rheumatoid arthritis. Clin Exp Immunol. 2013;173:76-83.

[34] Jo BS, Choi SS. Introns: The functional benefits of introns in genomes. Genomics Inform. 2015;13:112-8. 
[35] Nylander PO, Beckman L, Holmgren G, et al. Association of C3 and C4A complement types with familial amyloidotic polyneuropathy. Hum Hered. 1990;40:272-7.

[36] Zanusso GL, Moretto G, Bonetti B, et al. Complement neoantigen and vitronectin are components of plaques in amyloid AL neuropathy. Ital J Neurol Sci. 1992;13:493-9.

[37] Rafiq S, Frayling TM, Vyse TJ, et al. Assessing association of common variation in the C1Q gene cluster with systemic lupus erythematosus. Clin Exp Immunol. 2010;161:284-9.

[38] Liphaus BL, Umetsu N, Jesus AA, et al. Molecular characterization of the complement $\mathrm{C} 1 \mathrm{q}, \mathrm{C} 2$ and $\mathrm{C} 4$ genes in Brazilian patients with juvenile systemic lupus erythematosus. Clinics. 2015;70:220-7.

\section{Tables}

Table 1: Initial symptoms and signs among Cypriot ATTRV30M patients.

\begin{tabular}{ccc} 
Initial symptoms & Number of Patients & \% \\
\hline "Sharp shooting" or "burning" pains & $34 / 46$ & $74 \%$ \\
Nausea, vomiting, early satiety & $16 / 46$ & $35 \%$ \\
Diarrhea & $5 / 46$ & $11 \%$ \\
Constipation & $19 / 46$ & $41 \%$ \\
Impotence & $6 / 46$ & $13 \%$ \\
Bladder disturbances & $10 / 46$ & $22 \%$ \\
Sweating abnormalities & $0 / 46$ & 0 \\
Musculoskeletal (charcot joint) & $1 / 46$ & $2 \%$ \\
Dissociated sensory loss & $44 / 46$ & $96 \%$ \\
Orthostatic hypotension & $8 / 46$ & $17 \%$ \\
Esophageal reflux & $2 / 46$ & $4 \%$ \\
Hydronephrosis & $2 / 46$ & $4 \%$ \\
Loss of sensation in the feet & $2 / 46$ & $4 \%$ \\
Bilateral median and ulnar neuropathy & $1 / 46$ & $2 \%$ \\
Numbness in the feet & $1 / 46$ & $2 \%$ \\
Painless burns in the feet & $1 / 46$ & $2 \%$ \\
\hline
\end{tabular}

Note: Some patients presented with more than one symptom at the same time. 


\section{Figure Legend:}

Figure 1. Penetrance and age of disease onset. (a) The risk of developing the disease is shown to increase along with age. (b) The age of onset for living ATTRV30M patients in 5 year intervals is presented here, whereas the mean age of onset was found to be $40.6 \pm 12(n=46)$. 
a

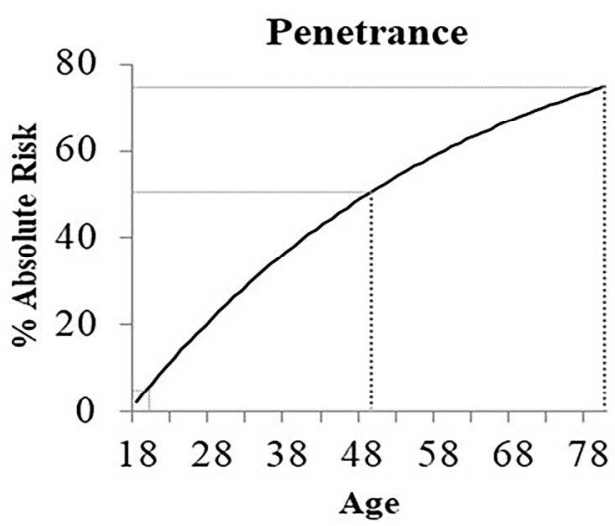

b Age of onset in living patients

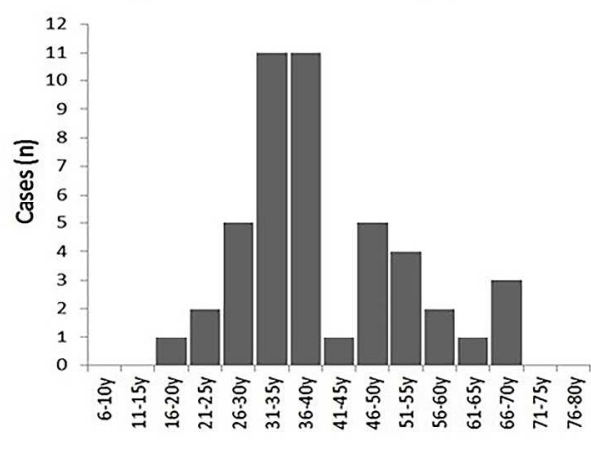

Figure 1. Penetrance and age of disease onset. (a) The risk of developing the disease is shown to increase along with age. (b) The age of onset for living ATTRV30M patients in 5 year intervals is presented here, whereas the mean age of onset was found to be $40.6 \pm 12(n=46)$.

$240 \times 100 \mathrm{~mm}(300 \times 300$ DPI) 
Supplementary Table 1: Minor allele frequency of all SNPs tested for cases and controls. Maf - minor allele frequency, Allele 1- minor allele/ allele 2- major allele *SNPs did not follow the Hardy-Weinberg equilibrium and were excluded from analyses.

\begin{tabular}{cccccc} 
SNP & Alleles & maf_cases & maf_controls & HWE_p_cases & HWE_p_controls \\
\hline rs172378 & G/A & 0.38 & 0.38 & 0.86 & 0.10 \\
rs9434 & A/C & 0.40 & 0.35 & 0.40 & 0.38 \\
rs12126436 & $\mathrm{A} / \mathrm{G}$ & 0.18 & 0.20 & 0.22 & 0.92 \\
rs209698 & $\mathrm{G} / \mathrm{A}$ & 0.45 & 0.40 & 0.06 & 0.35 \\
rs12128546 & $\mathrm{G} / \mathrm{T}$ & 0.27 & 0.25 & 0.31 & 0.87 \\
rs186037* & $\mathrm{G} / \mathrm{A}$ & 0.20 & 0.25 & 0.21 & 0 \\
rs158761 & $\mathrm{A} / \mathrm{C}$ & 0.39 & 0.38 & 0.92 & 0.05 \\
rs665691 & $\mathrm{G} / \mathrm{C}$ & 0.38 & 0.38 & 0.44 & 0.06 \\
rs6690827 & $\mathrm{A} / \mathrm{G}$ & 0.29 & 0.28 & 0.38 & 0.30 \\
rs12404537 & $\mathrm{T} / \mathrm{C}$ & 0.21 & 0.17 & 0.02 & 0.86 \\
rs672693 & $\mathrm{A} / \mathrm{G}$ & 0.38 & 0.33 & 0.09 & 0.05 \\
rs294180 & $\mathrm{A} / \mathrm{C}$ & 0.41 & 0.35 & 0.31 & 0.25 \\
rs12040131 & $\mathrm{G} / \mathrm{C}$ & 0.19 & 0.16 & 0.04 & 0.66 \\
rs7549747 & $\mathrm{C} / \mathrm{T}$ & 0.44 & 0.34 & 0.75 & 0.05 \\
rs7549888 & $\mathrm{T} / \mathrm{G}$ & 0.46 & 0.47 & 0.62 & 0.35 \\
rs17486657 & $\mathrm{T} / \mathrm{C}$ & 0.21 & 0.29 & 0.68 & 0.55 \\
rs294222 & $\mathrm{C} / \mathrm{G}$ & 0.34 & 0.37 & 0.96 & 0.35 \\
rs294223* & $\mathrm{T} / \mathrm{C}$ & 0.22 & 0.30 & 0.97 & 0.04 \\
rs17433871 & $\mathrm{C} / \mathrm{T}$ & 0.26 & 0.22 & 0.46 & 0.07 \\
rs15940 & $\mathrm{T} / \mathrm{C}$ & 0.32 & 0.22 & 0.56 & 0.22 \\
rs17433222 & $\mathrm{A} / \mathrm{G}$ & 0.24 & 0.22 & 0.09 & 0.84 \\
\hline
\end{tabular}


Supplementary Table 2: Estimated hazard ratios for earlier age of onset using survival analysis. Statistical analysis 1 includes both patients and asymptomatic carriers. Statistical analysis 2 includes patients only. Estimated SNPs hazard risk ratios. Statistical analysis 1- 99 ATTRV30M carriers (19 non-manifesting carriers /80 cases), Statistical analysis 2- 80 ATTRV30M cases. HR - Hazard Ratio / 95\% CI 95\% Confidence Interval

\begin{tabular}{c|cc|cc} 
& \multicolumn{2}{|c|}{$\begin{array}{c}\text { Statistical analysis No.1 } \\
\text { (99 ATTRV30M carriers) }\end{array}$} & \multicolumn{2}{c}{$\begin{array}{c}\text { Statistical analysis No. 2 } \\
\text { (80 ATTRV30M cases) }\end{array}$} \\
\hline SNP & HR (95\%CI) & P-value & HR (95\%CI) & P-value \\
rs172378 & $1.33(0.95-1.88)$ & 0.10 & $1.51(1.15-1.99)$ & $2.81 \times 10^{-3}$ \\
rs9434 & $1.53(1.10-2.14)$ & 0.01 & $1.44(1.05-1.97)$ & 0.02 \\
rs12126436 & $1.52(1.06-2.18)$ & 0.02 & $1.51(1.01-2.26)$ & 0.05 \\
rs209698 & $1.26(0.96-1.66)$ & 0.10 & $1.19(0.89-1.59)$ & 0.24 \\
rs12128546 & $1.37(0.95-1.97)$ & 0.09 & $1.34(0.94-1.92)$ & 0.11 \\
rs158761 & $1.28(0.92-1.79)$ & 0.14 & $1.53(1.16-2.00)$ & $2.24 \times 10^{-3}$ \\
rs665691 & $1.45(1.07-1.98)$ & 0.02 & $\mathbf{1 . 5 4}(\mathbf{1 . 1 7 - 2 . 0 3 )}$ & $\mathbf{1 . 8 7 \times 1 0 ^ { - 3 }}$ \\
rs6690827 & $1.28(0.90-1.81)$ & 0.16 & $1.26(0.89-1.79)$ & 0.19 \\
rs12404537 & $1.29(0.86-1.93)$ & 0.22 & $1.18(0.76-1.85)$ & 0.46 \\
rs672693 & $\mathbf{1 . 6 3}(\mathbf{1 . 1 5 - 2 . 3 0 )}$ & $\mathbf{5 . 8 7 x 1 0}$ & $1.54(1.10-2.16)$ & 0.01 \\
rs294180 & $1.51(1.08-2.10)$ & 0.01 & $1.43(1.04-1.95)$ & 0.03 \\
rs12040131 & $1.55(0.87-2.74)$ & 0.13 & $1.42(0.86-2.36)$ & 0.17 \\
rs7549747 & $1.26(0.89-1.79)$ & 0.19 & $1.53(1.10-2.13)$ & 0.01 \\
rs7549888 & $1.11(0.84-1.47)$ & 0.45 & $1.07(0.74-1.55)$ & 0.71 \\
rs17486657 & $0.92(0.61-1.37)$ & 0.67 & $0.90(0.57-1.43)$ & 0.66 \\
rs294222 & $0.76(0.53-1.11)$ & 0.15 & $0.68(0.48-0.95)$ & 0.03 \\
rs17433871 & $1.13(0.78-1.64)$ & 0.51 & $1.09(0.79-1.51)$ & 0.58 \\
rs15940 & $1.34(0.91-1.96)$ & 0.14 & $1.25(0.94-1.65)$ & 0.13 \\
rs17433222 & $1.23(0.78-1.94)$ & 0.38 & $1.31(0.87-1.97)$ & 0.20 \\
\hline
\end{tabular}


Supplementary Table 3: The squared correlation coefficient between the tested SNPs in the carriers.

\begin{tabular}{|c|c|c|c|c|c|c|c|c|c|c|c|c|c|c|c|c|c|c|c|}
\hline SNP & 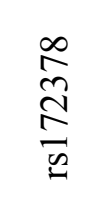 & 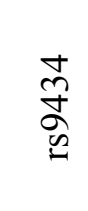 & 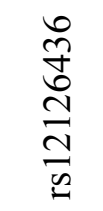 & 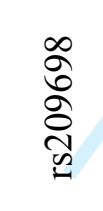 & 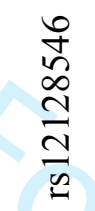 & $\begin{array}{l}\bar{b} \\
\infty \\
\infty \\
\stackrel{n}{n} \\
0\end{array}$ & 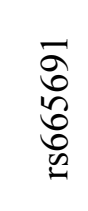 & $\begin{array}{l}\hat{\lambda} \\
\infty \\
0 \\
8 \\
0 \\
0 \\
0 \\
0\end{array}$ & 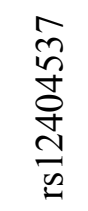 & 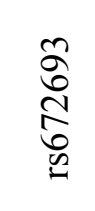 & 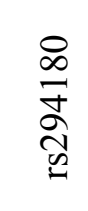 & 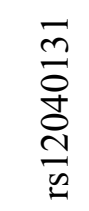 & 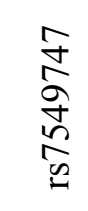 & $\begin{array}{l}\infty \\
\infty \\
\infty \\
\infty \\
\stackrel{+}{\infty} \\
\infty \\
\infty\end{array}$ & $\begin{array}{l}\hat{n} \\
0 \\
0 \\
\infty \\
\frac{0}{ \pm} \\
\bar{n}\end{array}$ & 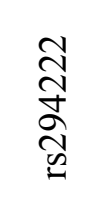 & 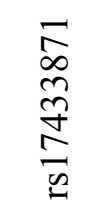 & $\begin{array}{l}\text { 온 } \\
\text { in } \\
\frac{n}{n}\end{array}$ & 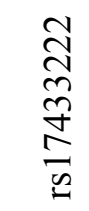 \\
\hline rs172378 & 1 & 0.36 & 0.34 & 0.38 & 0.25 & 0.76 & 0.81 & 0.66 & 0.43 & 0.30 & 0.36 & 0.27 & 040 & 0.09 & 0.02 & 0.28 & 0.19 & 0.23 & 0.40 \\
\hline rs9434 & 0 & 1 & & .28 & 43 & 0.32 & 0.33 & 0.42 & 0.44 & 0.86 & 0.98 & 0.36 & 0.26 & 0.30 & .01 & 0.37 & .15 & 0.58 & .50 \\
\hline rs12126436 & 34 & 0.29 & 1 & 0.38 & 0.66 & 0.28 & 0.30 & 0.38 & 0.64 & 0.29 & 0.28 & 0.22 & 0.07 & 0.16 & & 0.10 & .06 & 0.23 & 0.34 \\
\hline rs209698 & 0.38 & 0.28 & 0.38 & 1 & 0.53 & 0.44 & 0.45 & 0.19 & 0.34 & 0.32 & 0.28 & 0.18 & 0.20 & 0.06 & 0.01 & 0.16 & 0.07 & 0.21 & 0.25 \\
\hline rs12128546 & 0.25 & 0.43 & 0.66 & 0.53 & 1 & 0.23 & 0.24 & 0.32 & 0.44 & 0.46 & 0.44 & 0.20 & 0.12 & 0.17 & & 0.15 & 0.10 & 0.31 & 0.36 \\
\hline rs158761 & 0.76 & 0.32 & 0.28 & 0.44 & 0.23 & 1 & 0.92 & 0.46 & 0.38 & 0.39 & 0.33 & 0.21 & 0.29 & 0.12 & & 0.26 & 0.18 & 0.16 & 0.38 \\
\hline rs66569 & 81 & 0.33 & 0.30 & 0.45 & 0.24 & 0.92 & 1 & 0.50 & 0.41 & 0.41 & 0.34 & 0.22 & 0.28 & 0.13 & 0 & 0.26 & 0.19 & 0.15 & 0.38 \\
\hline rs6690827 & 66 & 42 & .38 & 0.19 & 0.32 & 0.46 & 0.50 & 1 & 0.57 & 0.35 & 0.43 & 0.36 & 0.27 & 0.18 & 0.0 & 0.19 & 0.31 & 0.31 & 0.54 \\
\hline rs 12404537 & 0.43 & 0.44 & 0.64 & 0.34 & 0.44 & 0.38 & 0.41 & 0.57 & 1 & 0.45 & 0.42 & 0.50 & 0.18 & 0.25 & 0 & 0.17 & 0.17 & 0.40 & 0.58 \\
\hline rs672693 & 0.30 & 0.86 & 0.29 & 0.32 & 0.46 & 0.39 & 0.41 & 0.35 & 0.45 & 1 & 0.88 & 0.35 & 0.21 & 0.33 & 0.01 & 0.32 & 0.15 & 0.46 & 0.54 \\
\hline rs294180 & 0.36 & 0.98 & 0.28 & 0.28 & 0.44 & 0.33 & 0.34 & 0.43 & 0.42 & 0.88 & & 0.34 & 0.26 & 0.31 & 0.01 & 0.38 & 0.16 & 0.56 & 0.51 \\
\hline & 0 & 0.36 & & 0.18 & 0.20 & 0.21 & 0.22 & 0.36 & 0.50 & 0.35 & 0.34 & 1 & 0.41 & 0.23 & & 0.19 & 0.39 & 0.26 & 0.62 \\
\hline rs7549747 & & & 0.07 & 0.20 & 0.12 & 0.29 & 0.28 & 0.27 & 0.18 & 0.21 & 0.26 & 0.41 & 1 & 0.03 & 0.19 & 0.43 & 0.29 & 0.23 & 0.31 \\
\hline rs7549888 & 0.09 & 0.30 & 0.16 & 0.06 & 0.17 & 0.12 & 0.13 & 0.18 & 0.25 & 0.33 & 0.31 & 0.23 & 0.03 & 1 & 0.35 & 0.48 & 0.14 & 0.13 & 0.26 \\
\hline rs17486657 & 0.02 & 0.01 & 0 & 0.01 & 0 & 0 & 0 & 0.01 & 0 & 0.01 & 0.01 & 0.08 & 0.19 & 0.35 & 1 & 0.14 & 0.05 & 0 & 0.03 \\
\hline rs294222 & 0.28 & 0.37 & 0.10 & 0.16 & 0.15 & 0.26 & 0.26 & 0.19 & 0.17 & 0.32 & 0.38 & 0.19 & 0.43 & 0.48 & 0.14 & 1 & 0.15 & 0.23 & 0.19 \\
\hline rs17433871 & & & & 0.07 & 0.10 & 0.18 & 0.19 & 0.31 & 0.17 & 0.15 & 0.16 & 0.39 & 0.29 & 0.14 & 0.05 & 0.15 & & 0.05 & 0.27 \\
\hline rs15940 & 0.23 & 0.58 & 0.23 & 0.21 & 0.31 & 0.16 & 0.15 & 0.31 & 0.40 & 0.46 & 0.56 & 0.26 & 0.23 & 0.13 & 0 & 0.23 & 0.05 & 1 & 0.31 \\
\hline rs17433222 & 0.40 & 0.50 & 0.34 & 0.25 & 0.36 & 0.38 & 0.38 & 0.54 & 0.58 & 0.54 & 0.51 & 0.62 & 0.31 & 0.26 & 0.03 & 0.19 & 0.27 & 0.31 & 1 \\
\hline
\end{tabular}

